\title{
Application of an ex-vivo drug sensitivity platform towards achieving complete remission in a refractory T-cell lymphoma
}

\author{
Sanjay de Mel', Masturah B. M. Rashid², Xi Yun Zhang³, Jasmine Goh³, Chun Tsu Lee', Li Mei Poon', Esther H. L. Chan', \\ Xin Liu', Wee Joo Chng ${ }^{1,3}$, Yen Lin Chee', Joanne Lee ${ }^{1}$, Yi Ching Yuen', Jing Quan Lim², Burton K. H. Chia ${ }^{4}$, \\ Yurike Laurensia ${ }^{4}$, DaChuan Huang (14), Wan Lu Pang ${ }^{4}$, Daryl Ming Zhe Cheah ${ }^{4}$, Esther Kam Yin Wong ${ }^{4}$, \\ Choon Kiat Ong $\mathbb{1}^{4}$, Tiffany Tang ${ }^{5}$, Soon Thye Lim ${ }^{5}$, Siok Bian Ng ${ }^{3,6}$, Soo Yong Tan ${ }^{7}$, Hoi-Yin Loi ${ }^{8}$, Lip Kun Tan', \\ Edward K. Chow ${ }^{3,9,10}$ and Anand D. Jeyasekharan (1) ${ }^{1,3}$
}

\section{Dear Editor,}

Currently, there are no clinically approved methods for predicting the relative efficacy of drug combinations for individual patients with cancer. Ex-vivo drug sensitivity experiments with patient-derived tumor material potentially offers a solution to identifying appropriate combinations of therapies for individual patients ${ }^{1}$. However, such assays are typically limited by the quantity available for combinatorial analysis of multiple drugs. We recently developed an experimental-analytical hybrid method, Quadratic phenotypic optimization platform (QPOP), which ranks drug combinations using a limited amount of tumor ${ }^{2}$. QPOP identifies optimal combinations based on the observation that biological response to perturbations (such as therapeutic intervention) can be mapped to a second-order polynomial equation ${ }^{3}$. In our initial study, QPOP identified novel therapeutic combinations for drug-resistant multiple myeloma, using ex-vivo testing on primary tumor samples. However, the concordance of QPOP-based drug sensitivity prediction with actual patient response to treatment was not explored in that study. We now present a case illustrating the setup and utilization of a QPOP protocol as a clinical decision aid, to

\footnotetext{
Correspondence: Yen Lin Chee (yen_lin_chee@nuhs.edu.sg) or

Joanne Lee (joanne_lee@nuhs.edu.sg) or Edward K. Chow (csikce@nus.edu.sg) or Anand D. Jeyasekharan (csiadj@nus.edu.sg)

${ }^{1}$ Department of Haematology-Oncology, National University Hospital,

Singapore, Singapore

${ }^{2}$ KYAN Therapeutics, Singapore, Singapore

Full list of author information is available at the end of the article.

These authors contributed equally: Lip Kun Tan, Edward K. Chow, Anand D.

Jeyasekharan
}

identify an optimal salvage regimen for a patient with refractory lymphoma.

A 55-year-old male presented to the Hematology Department at the National University Hospital (NUH) Singapore for a second opinion with a refractory lymphoma. He was initially diagnosed and treated in his home country for NK/T-cell lymphoma/leukemia with SMILE (steroid, methotrexate, L-asparaginase, etoposide) and subsequently GDP (gemcitabine, cisplatin, and dexamethasone). His disease progressed rapidly after both these regimens, prompting him to travel to our center for further evaluation. On presentation at $\mathrm{NUH}$, the patient had weight loss, massive splenomegaly, anemia (hemoglobin $8.6 \mathrm{~g} / \mathrm{dl})$, thrombocytopenia $\left(25 \times 10^{9} / \mathrm{L}\right)$, and lymphocytosis (absolute lymphocyte count $65 \times 10^{9} / \mathrm{L}$ ). A computed tomography (CT) scan revealed massive splenomegaly with no evidence of lymphadenopathy elsewhere. A bone marrow aspirate (BMA) showed infiltration with atypical lymphoid cells comprising $89 \%$ of nucleated cells. Flow cytometry (FC) immunophenotyping of the malignant cells identified an aberrant CD3-/CD7 +/CD8dim/CD4-/CD16+/CD56+/CD2heterogeneous phenotype. The bone marrow trephine biopsy showed a lymphoid infiltrate featuring small lymphocytes with condensed chromatin and inconspicuous nucleoli with a sinusoidal pattern of infiltration (Fig. S1). Immunohistochemistry (IHC) showed positive expression of CD2 and CD7 (Fig. S1), but negativity for CD3 and Epstein Barr Virus encoded RNA. CD56 was weakly positive while Tcell receptor (TCR) gamma was expressed. Based on the clinical features, bone marrow morphology, IHC and FC 
phenotype, the diagnosis was revised to Hepatosplenic Tcell Lymphoma (HSTCL). HSTCL is a rare subtype of peripheral T-cell lymphoma (PTCL) with gamma-delta neoplastic T-cells, and a typical clinical presentation of hepatosplenomegaly, B-symptoms, and cytopenias due to bone marrow (BM) involvement ${ }^{4}$. Patients with HSTCL have an extremely poor outcome, with five-year survival rates of less than $10 \%^{5}$.

He was initially treated at our center with the B arm of the HyperCVAD regimen, which the disease was refractory to. This was followed by treatments with pembrolizumab, GVD (gemcitabine, vinorelbine, liposomal doxorubicin) and pralatrexate. All of these had virtually no effect on his disease, and his peripheral white blood count rose to $200 \times 10^{9} / \mathrm{L}$, with worsening splenomegaly and B-symptoms. During this period, the patient was also recruited onto a translational research protocol; domain specific review board (DSRB) 2017/00507, for the development of ex-vivo drug sensitivity testing in lymphoma. Details of the protocol and methods are described in the supplementary information. Briefly, in this case, the tumor sample was collected using standard venesection, and mononuclear cells were isolated from $10 \mathrm{ml}$ of a patient blood sample for short-term culturing of primary HSTCL. Combinatorial drug sensitivity analysis was performed by the QPOP method (Fig. S2), where the drug candidates comprised of standard regimens and active agents for T-cell lymphoma, chosen in consideration of the treatment history of the patient (Table S1). An orthogonal array composite design (OACD) was used to minimize the number of combinations for factor screening and in-depth analyses, in concentrations that represent clinically approved doses ${ }^{6}$. After 48-h drug treatment, the CellTiter-Glo ${ }^{\circledR}$ Luminescent Cell Viability Assay was used to quantify cell viability.

Eleven drugs at three doses were used in the initial QPOP experiment on the patient's sample. All possible permutations of these drugs were ranked based on a coefficient-specific quadratic function. Most conventional drug combinations ranked relatively low in the QPOP assay. For example; SMILE was ranked 3951 out of 14,784 possible 5-drug combinations, while GDP was ranked 493 out of 1320 possible 3-drug combinations (Tables S2 and S3, Fig. S3). Among all possible 3-drug sets, a combination of Cisplatin, Cytarabine, and L-asparaginase was predicted to be most efficacious for this patient's sample, which could potentially indicate utility of the DECAL regimen ${ }^{7}$. This pediatric regimen, however, is not a standard adult treatment protocol. Ex vivo sensitivity to pralatrexate as a single-drug was also evaluated by dose-response analysis, as the patient was planned for treatment with pralatrexate at the time of sample collection. The patient sample was largely unresponsive to pralatrexate (Fig. 1a). The in-vitro analyses of GDP and pralatrexate were concordant with the refractory responses observed in the patient following treatment with these regimens.

However, we noted a significant efficacy for bortezomib as monotherapy in the sample, highlighting the ability of QPOP to identify both mono- and combinatorial therapy from a single experiment. The dual treatment of bortezomib and a histone deacetylase inhibitor, panobinostat, was previously reported to be efficacious in a Phase II trial on relapsed or refractory PTCL patients, although HSTCL cases were not included ${ }^{8}$. Therefore, a dose-response analysis was performed on the patient sample for bortezomib and panobinostat, in isolation and in-combination (Fig. 1a). There was a significant shift in the dose-response curves when the drugs were administered in combination (Fig. 1a(i)), with combination indices of $<1$ across different concentrations: indicative of a synergistic interaction (Fig. $1 \mathrm{a}(\mathrm{ii})$ ). Integrating this serial dose-response dataset into QPOP analytics, we cross-compared the bortezomibpanobinostat combination with the predicted output of other regimens from the initial analysis (Fig. 1b). Response surface maps of bortezomib and panobinostat revealed a sharp decrease in viability as the concentrations increase, suggesting a highly synergistic interaction between bortezomib and panobinostat. As such, it was predicted that the patient would respond better to a combination of bortezomib-panobinostat (BP) than to the other drug combinations evaluated in the assay. Our protocol allows for the sharing of drug sensitivity analysis with the treating physician, and for subsequent genomic analysis of the tumor (DSRB study 2015/00176).

This patient had personal funds to cover the costs of off-label use of novel anti-cancer agents. Given the exquisite ex-vivo sensitivity noted to the BP combination, the lack of any standard treatment options, and the availability of a protocol for BP as described by Tan et al. ${ }^{8}$, a shared decision was made with the patient to initiate treatment with panobinostat $20 \mathrm{mg}$ three times per week and bortezomib $1.3 \mathrm{mg} / \mathrm{m}^{2}$ intravenously twice per week. After two cycles of BP, we saw a dramatic reduction in his lymphocytosis (Fig. 2a) The patient was in complete metabolic remission based on a positron emission tomography scan after 8 cycles of BP. (Fig. 2b, c). A bone marrow study showed no evidence of residual disease by morphology or FC. The patient underwent an autologous stem cell transplant (AutoSCT) as he had no matched donor for allogeneic transplant (AlloSCT) and reported wellness 1 year later. Complete remissions (CR) in HSTCL are rare, even more so after salvage therapy in refractory disease ${ }^{5}$. This represents, therefore, a unique case of HSCTL achieving CR with a bortezomibpanobinostat combination identified through ex-vivo drug sensitivity testing. Bortezomib has shown in vitro synergism with histone deacetylase (HDAC) inhibitors against $\mathrm{T}$-cell lymphoma cells ${ }^{9,10}$. However, molecular 
A

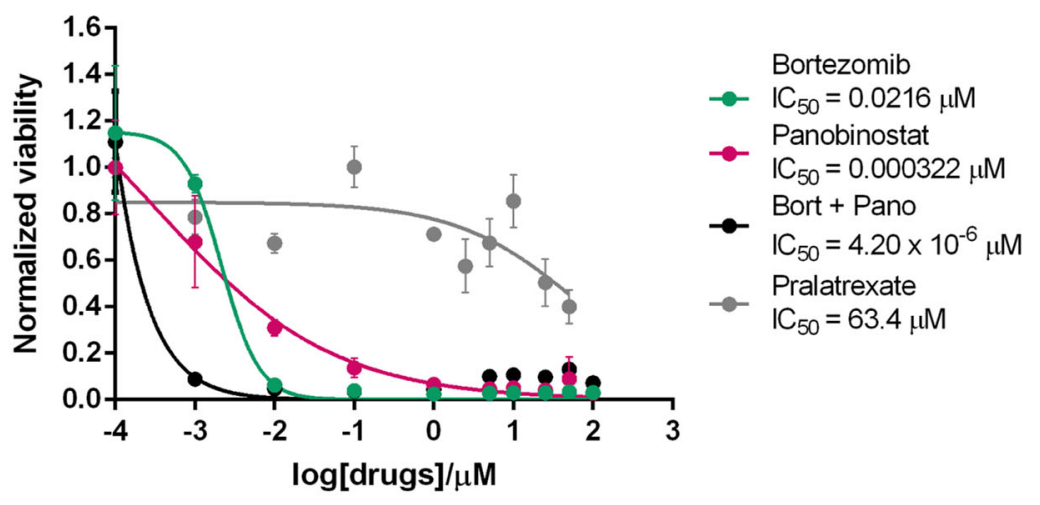

ii

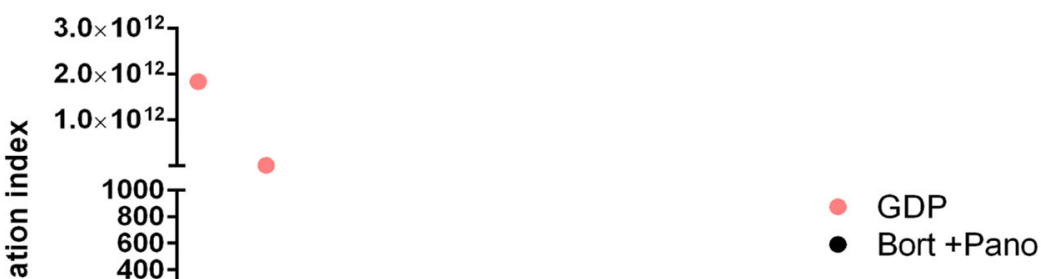

B
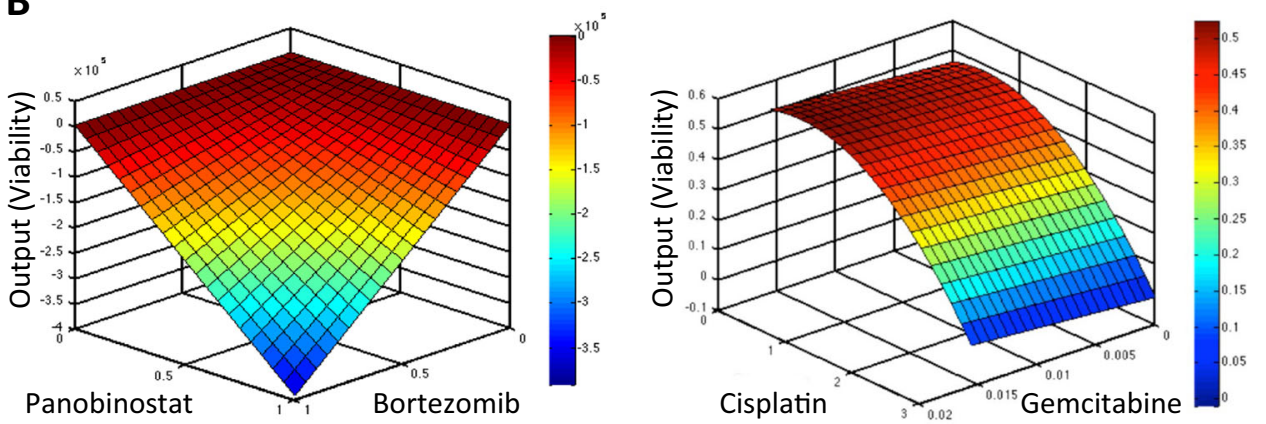

Fig. 1 Ex vivo drug sensitivity results. Ex vivo synergistic interaction of combinatorial therapy BP, compared to chemotherapy (a(i)) dose-response curve of bortezomib, panobinostat and pralatrexate as single agents, and the BP combination. The IC $\mathrm{C}_{50} \mathrm{~S}$ were $0.0216 \mu \mathrm{M}, 0.000322 \mu \mathrm{M}$ and $63.4 \mu \mathrm{M}$ for single agent bortezomib, panobinostat, and pralatrexate respectively. (a(ii)) combination indices of BP in relation to GDP. Data are presented as means \pm SD of three technical replicates. b Response surface maps illustrating the interaction between BP (left panel) and Gem-Cis (right panel).

markers of BP response are unknown. A comprehensive genomic and transcriptomic analysis was performed and is provided here (Tables S4 and S5, Fig. S4, EGA accession number: EGAD00001005229) to facilitate further studies into the molecular basis of this exceptional response in a typically fatal malignancy.

Ex-vivo drug sensitivity testing platforms using primary tumor samples hold the promise of identifying appropriate therapies for specific patients ${ }^{1,11,12}$. Previously published platforms rely on comparative single-drug or pairwise-drug sensitivity from multiple tumor samples, sometimes combined with large scale genomic analysis to build pharmacogenomic models ${ }^{13,14}$. We have previously demonstrated that QPOP rationally identifies optimal drug combinations in a sample-specific manner in myeloma cell lines and ex-vivo primary myeloma cells ${ }^{15}$. By applying a QPOP-based protocol towards ex-vivo drug combination sensitivity analysis of primary HSTCL cells 


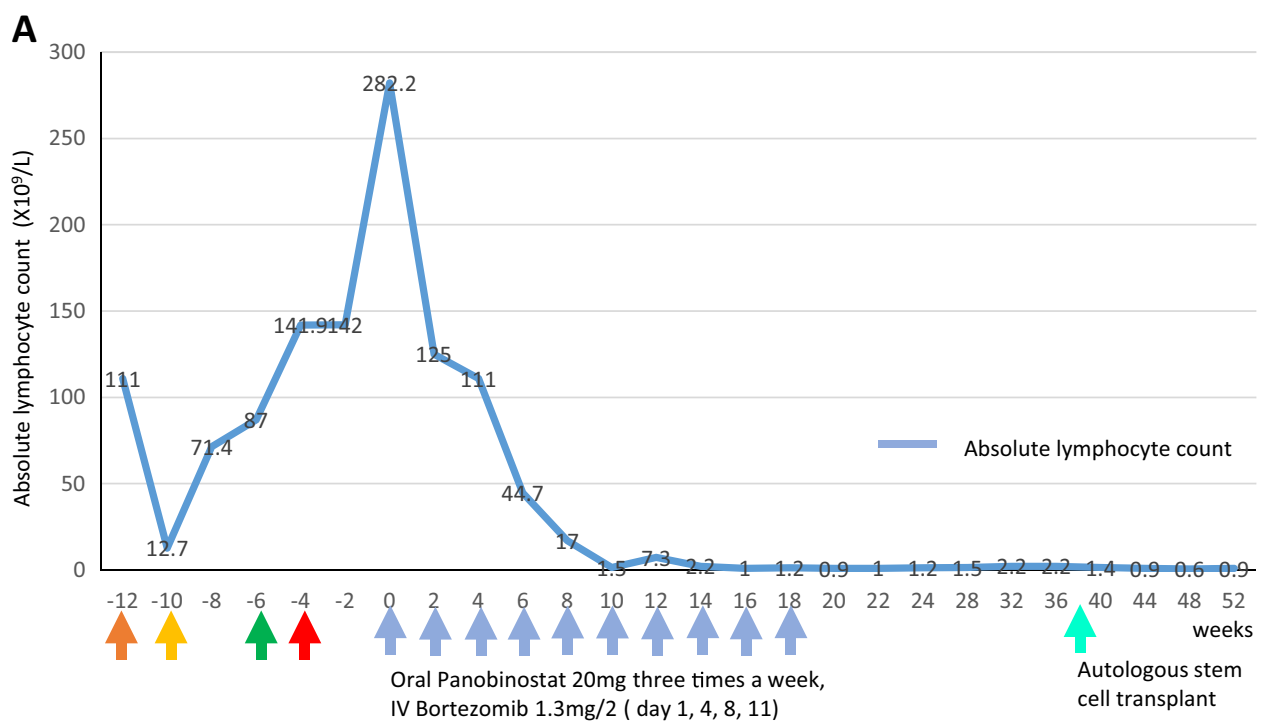

B
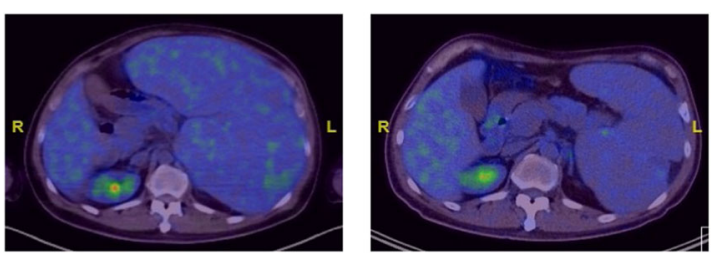

C

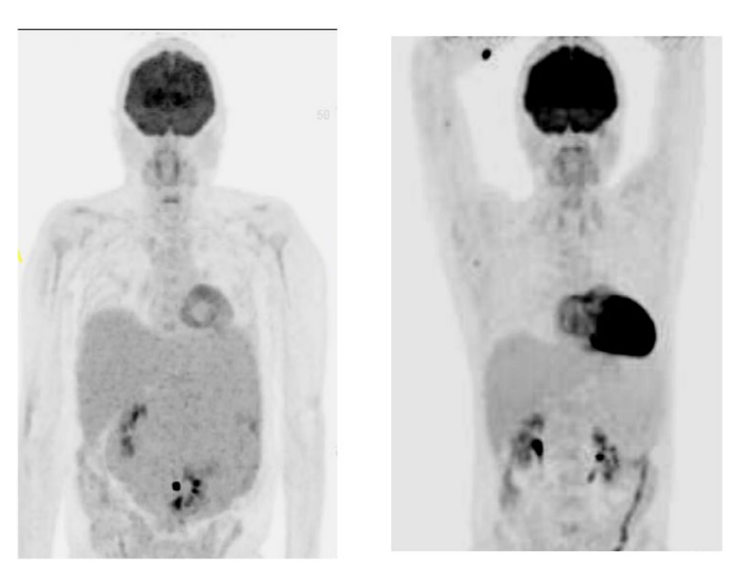

Fig. 2 Clinical Response to Treatment with BP. a The trend of the absolute lymphocyte count after treatment with each regimen. Color-coded arrows indicate treatment regimens given before bortezomib-panobinostat (blue arrows). Orange: HyperCVAD B, yellow: pembrolizumab, green: gemcitabine, vinorelbine, liposomal doxorubicin, red: pralatrexate. A rapid and sustained reduction of the ALC was seen after treatment with the BP regimen. This was accompanied by an improvement in hemoglobin and platelet count (data not shown). PET scan. $\mathbf{b}$ The post treatment scan shows an interval significant reduction in size of splenomegaly, measuring $15.6 \mathrm{~cm}$ craniocaudally compared with $27.9 \mathrm{~cm}$ at initial presentation. c The splenic FDG uptake has normalized in the interim. The previous scan shows heterogeneous increased FDG accumulation indicating disease involvement.

from the patient described in this paper, an actionable drug combination in bortezomib and panobinostat was identified within one week of sample collection. Specific drug panels will need to be tested in a larger number of cases to validate the relationship between in-vitro and invivo potency of QPOP identified combinations. Ex-vivo strategies have limitations in that tumor cell kill is assessed in the absence of the microenvironment and at doses that may not be accurately reflective of in-vivo levels. However, the high concordance between the QPOP assay and the clinical response in our index case highlights the feasibility and rationale for prospective studies 
and clinical trials investigating this platform in relapsed/ refractory lymphoma.

\section{Acknowledgements}

The ex-vivo QPOP study for lymphoma was supported by a junior investigator grant to ADJ from the National University Cancer Institute of Singapore (NCIS). Work in A.D.J. and E.K.C.'s laboratories are funded through the National Research Foundation Singapore and the Singapore Ministry of Education under its Research Centers of Excellence initiative. The sequencing of the case and bioinformatic analyses (OCK/LST lab) were supported by a National Medical Research Council collaborative grant (TCR) on Lymphoma, Tanoto Foundation Professorship in Medical Oncology and Singapore National Cancer Centre Research Fund. A.D.J. is a recipient of the Singapore Ministry of Health's National Medical Research Council Transition Award (NMRC/TA/0052/2016). This research is supported by Singapore Ministry of Health's National Medical Research Council under its Open Fund-Large Collaborative Grant ("OF-LCG") (MOH-OFLCG18May-0028).

\section{Author details}

'Department of Haematology-Oncology, National University Hospital, Singapore, Singapore. ${ }^{2}$ KYAN Therapeutics, Singapore, Singapore. ${ }^{3}$ Cancer Science Institute of Singapore, Yong Loo Lin School of Medicine, National University of Singapore, Singapore, Singapore. ${ }^{4}$ Division of Cellular and Molecular Research, National Cancer Centre Singapore, Singapore, Singapore. ${ }^{5}$ Division of Medical Oncology, National Cancer Centre Singapore, Singapore, Singapore. ${ }^{6}$ Department of Pathology, Yong Loo Lin School of Medicine, National University of Singapore, Singapore, Singapore. 'Department of Pathology, National University Hospital, Singapore, Singapore. ${ }^{8}$ Department of Diagnostic Imaging, National University Hospital Singapore, Singapore, Singapore. 'Department of Pharmacology, Yong Loo Lin School of Medicine, National University of Singapore, Singapore, Singapore. ${ }^{10} \mathrm{~N} .1$ Institute for Health, National University of Singapore, Singapore, Singapore

\section{Author contributions}

Experimental design: E.K.C., M.B.R., X.Y.Z., S.D.M. and A.D.J. QPOP experiments and analyses: E.K.C., M.B.R., X.Y.Z. and J.G. Pathological and radiology review: S.Y.T., S.B.N. (Pathology), H.Y.L. (Radiology). Sequencing and Bioinformatic analysis: J.Q.L., B.K.H.C., Y.L., C.K.O. Coordination of sample provision/ethics approvals: X.L. Writing of the paper: S.D.M., M.B.M.R., C.T.L., L.J.Q., B.C.K.H., Y.L., X.Y.Z., E.K.C. and A.D.J. Critical Review and Editing of the paper: M.L.M.P., W.J.C., C.K.O., T.T., L.S.T., S.B.N., S.Y.T., L.K.T., E.K.C. and A.D.J.

\section{Conflict of interest}

A.D.J.: honoraria from AstraZeneca, Janssen, and MSD, travel funding from Perkin Elmer, and research funding from Janssen. E.C.; shareholder in KYAN Therapeutics. The other co-authors declare no relevant conflict of interest for this work.

\section{Publisher's note}

Springer Nature remains neutral with regard to jurisdictional claims in published maps and institutional affiliations.
Supplementary Information accompanies this paper at (https://doi.org/ 10.1038/s41408-020-0276-7).

Received: 5 November 2019 Revised: 5 January 2020 Accepted: 10 January 2020

Published online: 27 January 2020

\section{References}

1. Chia, S. et al. Phenotype-driven precision oncology as a guide for clinical decisions one patient at a time. Nat Commun. 8, 435 (2017).

2. Rashid, M. et al. Optimizing drug combinations against multiple myeloma using a quadratic phenotypic optimization platform (QPOP). Sci. Transl. Med. https://doi.org/10.1126/scitranslmed.aan0941 (2018).

3. Al-Shyoukh, I. et al. Systematic quantitative characterization of cellular responses induced by multiple signals. BMC Syst. Biol. 5, 88 (2011).

4. Belhadj, K. et al. Hepatosplenic gammadelta T-cell lymphoma is a rare clinicopathologic entity with poor outcome: report on a series of 21 patients. Blood 102, 4261-4269 (2003).

5. Ferreri, A. J., Govi, S. \& Pileri, S. A. Hepatosplenic gamma-delta T-cell lymphoma. Crit. Rev. Oncol. Hematol. 83, 283-292 (2012).

6. $\mathrm{Xu}, \mathrm{H}$., Jaynes, J. \& Ding, X. Combining two-level and three-level orthogonal arrays for factor screening and response surface exploration. Stat. Sinica $\mathbf{2 4 ,}$ 269-289 (2014)

7. Kobrinsky, N. L. et al. Outcomes of treatment of children and adolescents with recurrent non-Hodgkin's lymphoma and Hodgkin's disease with dexamethasone, etoposide, cisplatin, cytarabine, and l-asparaginase, maintenance chemotherapy, and transplantation: Children's Cancer Group Study CCG-5912. J. Clin. Oncol. 19, 2390-2396 (2001).

8. Tan, D. et al. Panobinostat in combination with bortezomib in patients with relapsed or refractory peripheral T-cell lymphoma: an open-label, multicentre phase 2 trial. Lancet Haematol. 2, e326-e333 (2015).

9. Heider, U. et al. Synergistic interaction of the histone deacetylase inhibitor SAHA with the proteasome inhibitor bortezomib in cutaneous T cell lymphoma. Eur. J. Haematol. 82, 440-449 (2009).

10. Zhang, Q. L. et al. The proteasome inhibitor bortezomib interacts synergistically with the histone deacetylase inhibitor suberoylanilide hydroxamic acid to induce T-leukemia/lymphoma cells apoptosis. Leukemia 23, 1507-1514 (2009).

11. Lee, D. W. et al. High-throughput screening (HTS) of anticancer drug efficacy on a micropillar/microwell chip platform. Anal. Chem. 86, 535-542 (2014).

12. Lee, J. K et al. Pharmacogenomic landscape of patient-derived tumor cells informs precision oncology therapy. Nat. Genet. 50, 1399-1411 (2018).

13. Frismantas, V. et al. Ex vivo drug response profiling detects recurrent sensitivity patterns in drug-resistant acute lymphoblastic leukemia. Blood 129, e26-e37 (2017).

14. Fischer, U. et al. Genomics and drug profiling of fatal TCF3-HLF-positive acute lymphoblastic leukemia identifies recurrent mutation patterns and therapeutic options. Nat. Genet. 47, 1020-1029 (2015).

15. Mohd Abdul Rashid, M. B. et al. Identification and optimization of combinatorial glucose metabolism inhibitors in hepatocellular carcinomas. J. Lab. Autom. 20, 423-437 (2015). 